\title{
Human performance on random ratio and random interval schedules, performance awareness and verbal instructions
}

\author{
Ceri A. Bradshaw ${ }^{1} \cdot$ Gary Freegard $^{1} \cdot$ Phil Reed $^{1}$
}

Published online: 8 April 2015

(C) Psychonomic Society, Inc. 2015

\begin{abstract}
Humans responded on multiple random-ratio (RR) random-interval (RI) schedules, and their verbalized performance awareness (PA; i.e., their ability to accurately describe what they did) was measured in three experiments. In Experiment 1, instructions informed participants that to earn points, either sometimes rapid responding and sometimes slow responding would work best (accurate instructions); rapid responding would work best (go fast instructions); spaced responding would work best (go slow instructions); or no advice was provided (minimal instructions). In Experiments 2 and 3, participants received either accurate or minimal instructions and were subject to extinction after a multiple RRRI schedule. In all experiments, both performance awareness, and receiving accurate instructions, were related to schedulesensitive responding, but were unrelated to one another - participants receiving accurate-rate instructions were not more likely to show performance awareness than those exposed to minimal instructions. Both higher performance awareness and exposure to accurate instructions predicted faster extinction in Experiment 2 but not in Experiment 3. The current results suggest that performance awareness rather than contingency awareness is more strongly related to humans displaying schedule-typical behavior and that this is not strongly related to any explicit verbal instructions that are given.
\end{abstract}

Keywords Schedule awareness $\cdot$ Instructions $\cdot$ Random ratio $\cdot$ Random interval $\cdot$ Schedules $\cdot$ Humans

Variable-ratio and variable-interval (or the functionally nearidentical random-ratio or random-interval) schedules produce

Phil Reed

p.reed@swansea.ac.uk

1 Department of Psychology, Swansea University, Singleton Park, Swansea SA2 8PP, UK consistent patterns of responding across many species: variable-ratio (VR) or random-ratio (RR) schedules generally supporting higher rates of response and variable-interval (VI) or random-interval (RI) schedules generally supporting lower response rates (see Catania, Matthews, Silverman, \& Yohalem, 1977; Catania \& Reynolds, 1968; Ferster \& Skinner, 1957; Peele, Casey, \& Silverberg, 1984; Reed, 2011; Reynolds, 1975; Zuriff, 1970). These differentiated VR-RR versus VI-RI response rates are also sometimes seen in humans (see Baron \& Galizio, 1983; Bradshaw \& Reed, 2012; Matthews, Shimoff, Catania, \& Sagvolden, 1977; Raia, Shillingford, Miller, \& Baier, 2000; Reed, 1993, 1994, 1999, 2001; Shimoff, Catania, \& Matthews, 1981). However, schedule-induced response rates in humans are far less consistent than those of nonhumans, and findings have suggested that a significant proportion of human participants perform in a manner which is insensitive to the contingency of the schedule presented (see Bradshaw \& Reed, 2012; Catania, Matthews, \& Shimoff, 1982; Matthews et al., 1977; Shimoff et al., 1981; Wearden \& Shimp, 1985).

The difference between human and nonhuman schedule response patterns may hold some significance in terms of understanding the underlying factors that control schedule behavior in humans (see Leander, Lippman, \& Meyer, 1968; Lippman \& Meyer, 1967; Lowe, 1979; Lowe, Harzem, \& Hughes, 1978; Weiner, 1964, 1969, 1970). At present, the reasons for contingency-sensitive versus contingencyinsensitive human responding on schedules are unclear, but differentiated patterns of responding have been linked to factors such as whether the reinforcer requires a consummatory response (Matthews et al., 1977), the type of reinforcement employed (Lowe, Harzem, \& Bagshaw, 1978), whether performance is shaped or instructed by experimenters (Catania et al., 1982; Matthews, Catania, \& Shimoff, 1985; Matthews et al., 1977; Shimoff et al., 1981; Shimoff, Matthews, \& Catania, 1986), and to the degree that the participants demonstrate contingency or performance awareness (Bradshaw \& 
Reed, 2012; Hayes, Brownstein, Zettle, Rosenfarb, \& Korn, 1986b; Wearden \& Shimp, 1985b). In relation to these latter two concepts, "contingency awareness" (CA) is taken to be the participant's ability to describe what relationship is actually required between response and outcome (e.g., Brewer, 1974; Lippman \& Meyer, 1967), whereas "performance awareness" (PA) is taken to be the participant's ability to accurately describe the behavior that he or she has just emitted (e.g., Bradshaw \& Reed, 2012). There is a complex pattern of results relating to the impact of CA and PA on human schedule behavior, the relationship between the two, and with attempts to manipulate them through the provision of verbal instructions, which is the focus of the current manuscript.

Evidence from research employing forms of conditioning other than operant schedules (e.g., classical conditioning) suggests that $\mathrm{CA}$ is an important factor in human conditioned responding. A number of studies have shown that awareness of the CS-UCS contingency in classical conditioning procedures is necessary for conditioned responding (see Brewer, 1974; Dawson \& Schell, 1985; Mitchell, De Houwer, \& Lovibond, 2009, for reviews of classical conditioning and contingency awareness). Evaluative conditioning (EC) research also supports a link between CA and conditioned responding (see Pleyers, Corneille, Luminet, \& Yzerbyt, 2007; Stahl \& Unkenbach, 2009; Stahl, Unkelbach, \& Corneille, 2009). Although this association has not consistently been demonstrated across all studies (cf. Baeyens, Eelen, \& Van den Bergh, 1990; Dickinson \& Brown, 2007; Walther \& Nagengast, 2006), CA has been suggested as an important moderator of $\mathrm{EC}$, with the effects of EC appearing more pronounced for higher, rather than lower, CA participants (Hofmann, De Houwer, Perugini, Baeyens, \& Crombez, 2010).

Operant conditioning studies have also reported a link between CA and human performance. For example, Lippman and Meyer (1967), and Leander et al. (1968), reported correlations between operant performance on fixed interval (FI) schedules and participant self-report of the conditioning contingencies. Wearden and Shimp (1985) measured knowledge of how reinforcement was being delivered in terms of participants' opinions of the best way to receive a reward for their responses, which can more accurately be described as a measure of the relationship between their own behavior and its outcome, or performance awareness (PA), and found that schedule-sensitive responding was reliably related to PA in experimental paradigms involving RI schedules.

Catania et al. (1982; see also Matthews et al., 1985; Shimoff et al., 1981) investigated the relationships between contingency-shaped versus instructed responding, awareness of performance and/or contingency, and "schedule sensitive" performance. They noted that low-rate responding, established by shaping, changed accordingly with changes of contingency, but that instructed responding did not alter in line with changes in the contingency. This finding suggested that shaping produces more contingency-sensitive responding than instructional control (Shimoff et al., 1981). However, neither shapednor instructed-responses produced consistent RR-RI rate differences. Shimoff et al. (1981) and Matthews et al. (1985) examined differences between schedule-sensitive performance and either PA or CA, and noted that performance was consistent with the participants' verbal descriptions of their behavior, even when response rates were opposite to that typically produced by the contingency. In contrast, when participants produced contingency descriptions, their performance was inconsistently related to their descriptions, and was sometimes schedule-sensitive and sometimes not.

Overall, the results of these studies suggest that, although the relationship between shaped responding and awareness of the schedule reinforcement paradigm is inconsistent, participants are generally more likely to respond in a contingencysensitive manner when their guesses corresponded with the correct contingency. They also suggest that performanceawareness measurement relates to greater consistency between descriptions and responding and more schedulesensitive responding than contingency-awareness measurement and, finally, that shaped behavior is more schedulesensitive than instructed behavior is. This summary is consistent with the role of verbalized performance awareness and its relationship with RR-RI schedule performance in humans demonstrated in an earlier study by Bradshaw and Reed (2012), where we demonstrated that higher PA was reliably associated with schedule-sensitive responding and that lower PA was associated with schedule-insensitive performance.

To measure the effect of instructions on human schedule performance, Hayes, Brownstein, Haas, and Greenway (1986a) examined schedule sensitivity through a multiple FR DRL schedule and by changing the operative schedule once consistent responding had been established. Participants received minimal instructions, instructions appropriate to ratio schedules only, instructions appropriate to interval schedules only, or instructions appropriate to both schedules. Once the participants were responding on the multiple schedule in a stable manner, they were placed into extinction. For participants who responded in a schedule-insensitive manner, there was little reduction in responding when the task no longer yielded reinforcement. Large extinction effects occurred for most of the participants who had been given inaccurate instructions but who had responded sensitively to the schedule. However, where accurate instructions had been given by experimenters, around half of the participants showed small extinction effects. The results suggest that extinction occurred more rapidly in those participants who showed self-generated sensitivity to schedule contingencies. Participants who were apparently schedule-insensitive, or for whom sensitivity had occurred due to experimenter instructions, continued to respond with few extinction effects being demonstrated (Hayes et al, 1986a). 
The results from both Catania et al. (1982) and Hayes et al. (1986a) suggest that contingency-sensitive responding can be elicited in some humans by either instructing participants or by shaping them but that neither method produces consistent schedule-sensitive responding across all participants. Furthermore, Hayes et al. (1986a) suggest that, where contingency-sensitive responding is seen, performance is far more robust if that responding has been self-generated, rather than instructed. Both studies also reported a link between sensitive responding and PA; however, the differences in procedure between these studies, and between these studies and our previous study (Bradshaw \& Reed, 2012), make comparisons between all the studies difficult. Furthermore, the studies by both Catania et al. (1982) and Hayes et al. (1986a) both use complex multiple schedules which also make comparisons to our work which involves a more straightforward procedure problematic.

In light of the apparent complexity in previous findings concerning sensitive schedule performance, PA, and verbal instructions (Catania et al., 1982; Hayes et al., 1986a; Hayes et al., 1986b; Matthews et al., 1985; Shimoff et al., 1981; Shimoff et al., 1986), the present set of studies aimed to explore these relationships further. In particular, it is hoped to establish whether CA or PA is most related to the emergence of schedule-typical responding in humans, whether either results in greater or lesser sensitivity to changes in the contingencies, and the relationship of both to the provision of explicit verbal instructions. Furthermore, where previous studies have often used complex tasks to investigate human performance (Catania et al., 1982; Hayes et al., 1986a; Hayes et al., 1986b; Matthews et al., 1985; Shimoff et al., 1986), the present set of studies used a simple RR-RI computer task in line with a previous investigation by Bradshaw and Reed (2012) to examine the role of verbalized performance awareness, verbal instructions given by experimenters, and schedule performance.

\section{Experiment 1}

In order to further explore this area, we felt it necessary to use a simple RR-RI paradigm to partially replicate procedural elements from the work by Catania et al. (1982), Hayes et al. (1986a), and Hayes et al. (1986b) and also to combine investigation of schedule performance, differentiated experimenter instructions, and PA, in one study. In doing so, we sought to clarify any relationships between these factors in a straightforward schedule task with humans. Thus, the aim of the first experiment was to combine the various elements of previous studies (i.e., Bradshaw \& Reed, 2012; Catania et al., 1982; Hayes et al., 1986a; Hayes et al., 1986b; Matthews et al., 1985; Shimoff et al., 1986) using a simple RR-RI task in order to examine whether sensitive schedule responding would show a relationship with performance awareness and/or verbal instructions. Furthermore, we aimed to examine whether either of these latter two factors would show a stronger relationship with sensitive schedule performance, and whether these two factors are related to one another.

\section{Method}

\section{Participants}

A sample of 35 students was recruited via the Psychology Department subject-pool system; they received credit for their participation, but no financial payment. The sample comprised 31 females and 4 males, aged between 18 and 23 years (mean $=19.43 \pm 1.07)$.

\section{Apparatus}

The experimental task was presented on a standard desktop computer. Visual Basic (6.0) was used to program the task, which consisted of two primary reinforcement schedule types. The program first presented a random-ratio (RR) schedule, wherein points, acting as reinforcers, were awarded for presses to the space bar according to a RR20 schedule (points were awarded after each space bar response with a $1 / 20$ probability). Participants also lost one point for each space bar response, regardless of whether the response was reinforced. This procedure was adopted as it has previously been established that the presence of such a response cost generates schedule performance by humans that is similar to that observed in nonhumans (see Raia et al., 2000). It has been argued that the absence of a response cost for a simple computer key press creates little reason to regulate performance in line with the contingency of the schedule, especially in contrast to effort needed for nonhumans to make a response in a conditioning chamber (see Bradshaw \& Reed, 2012; Reed, 2001). Each reinforcer consisted of 40 points being added to the participant's total. Second, an RI schedule was delivered, whereby 40 points were awarded following the first response after a specified amount of time had elapsed. The response cost contingency also applied to the RI schedule. The RI schedule was yoked to the preceding RR schedule, so that reinforcement in the RI schedule was delivered only after the elapse of time that it had taken for the corresponding reinforcer to be awarded on the RR trial.

The computer task presented a white screen, with a stimulus boxes placed in the center upper portion of the screen. The box was approximately 8 -cm wide $\times 3$-cm high, and was blocked with a single color (either red or green), to indicate schedule type. A new schedule was, thus, indicated by the 
changing color of this box. For the first trial (RR) it was red (for half the participants), followed by green for the second trial (RI), and alternated in this manner for the subsequent trials. For the other half of the participants the box was green for the first (RR) trial and red for the second (RI) trial, recurring. Participants were informed that the box would change color when a new trial commenced but were not informed of which schedule type the color indicated. Underneath the color stimulus box the word "POINTS" (in capital letters) was positioned, and below this the running total of the points in figures appeared. Points were set to 10 at the commencement of the task, and reduced by one with every space bar press. When reinforcement was delivered, points would rise by 40 .

\section{Materials}

A performance awareness questionnaire was administered to retrospectively measure the participants' awareness of the relationship between their behavior and reinforcement (through points awarded) in the task. This was previously used by Bradshaw and Reed (2012), and was based on the questions used by Catania et al. (1982; see also Matthews et al., 1985; Shimoff et al., 1986), to assess performance awareness, with the notable difference that participants in the aforementioned research were required to fill in the answer to the question in their own words. In the present research, the question posed in each of the questionnaire items was based on the questions asked in the research cited above, but the participants were required to indicate their answer in terms of one closed response from six options presented for each question. In total eight questions were asked, each related on one of the eight trials within the task, and each item asked the same question:

In the [first/second] game in the task, what did you consider to be the best way to increase your points score?:

(a) Press space bar very little; (b) Do not press space bar at all; (c) Press space bar a lot in a small amount of time;

(d) Pause in between space bar presses; (e) Press space bar a little in a small amount of time; (f) I do not know.

For RR trials, the correct response was deemed to be option (c) "Press space bar a lot in a small amount of time." If participants indicated this response for the items related to RR trials $(1,3,5$, and 7) they received a score of 2 points for each correct response (see Bradshaw \& Reed, 2012: Catania et al., 1982; Matthews et al., 1985; Shimoff et al., 1986). For RI trials, the correct response was considered to be option (e) "Press space bar a little in a small amount of time." Participants were awarded 2 points each time they indicated this response in relation to RI trials $(2,4,6$, and 8; see Bradshaw \& Reed, 2012; Catania et al., 1982; Matthews et al.,
1985; Shimoff et al., 1986). Any other responses indicated received a score of zero. Thus, the maximum score it was possible to be awarded for performance awareness across all eight trials was 16 .

\section{Procedure}

Participants were tested individually in a quiet room, which contained a desk and computer, with the monitor situated approximately $60 \mathrm{~cm}$ from them. Participants gave written consent, and read the study information and paper instructions for the task. Participants commenced the task in their own time, and were required to fill in basic demographic details about themselves before the schedule task was presented.

Each schedule presentation was 4 min long, and a RR schedule trial was always presented immediately prior to the yoked RI schedule trial. There were four presentations of the yoked RR-RI pairs. The procedure of yoking RI trials to preceding RR trials ensured that reinforcement in the RI schedule was delivered after a similar elapse of time that it had taken for the corresponding reinforcer to be awarded on the RR trial.

Participants were randomly allocated to one of four groups: Minimal instructions, Go Fast, Go Slow, or Accurate Rate instructions (after Hayes et al., 1986a; Hayes et al. 1986b). Prior to the task beginning, all participants were presented with instructions on the computer screen, with variation according to experimental condition. All participants were presented with the following instructions:

When the task begins, use the space bar to score as many points as possible. There are eight games in total. The first game is identified with a large red [green] rectangle at the top of the screen. When the first game is over, the rectangle will change to green [red] to indicate the start of the next game. The rectangles alternate between red and green [green and red] to indicate the changing games for the remainder of the task. Your goal in each game is to reach the highest score possible. You will see that the points reduce according to the way in which you play, but will rise again every so often, according to the pattern of space bar hits that you use. All you need to do is to find the best pattern of space bar hits to score as highly as possible in each game. It may be a good idea to respond quickly sometimes and slowly at other times, but you need to discover this for yourself!

In addition to these, a further set of instructions appeared to participants on a further screen. The Minimal group was told:

Remember, your task is to score as many points as possible by pushing the space bar. 
The Go Fast group was told:

Remember, your task is to score as many points as possible by pushing the space bar. Rapid pushes on the space bar will work best.

The go Slow Group was told:

Remember, your task is to score as many points as possible by pushing the space bar. Pushes on the space bar with several seconds in between them will work best.

The Accurate Rate group was told (after Hayes et al., 1986a):

Remember, your task is to score as many points as possible by pushing the space bar. The rectangle which changes color on the trials is important. When it is one color, rapid pushes on the space bar will work best. When it is the other color, pushes with several seconds in between them will work best.

The participants were then instructed to click a start button to continue with the experiment. After task completion, participants completed the performance awareness questionnaire before leaving the laboratory.

\section{Results and discussion}

\section{Experimenter instructions}

Figure 1 presents the response rates for each exposure to each of the two schedule types (RR and RI), for the four groups of participants (Minimal, Go Fast, Go Slow, and Accurate Rate instructions). A three-factor, mixed-model analysis of variance (ANOVA) with instruction group as a between-subject factor, and schedule and trial as within-subject factors, was conducted on the data shown in Figure 1. This analysis revealed statistically significant main effects of schedule, $F(1$, $31)=29.84, p<.001$, Partial $\mathrm{Eta}^{2}=.49$, and instruction group, $F(3,31)=4.66, p<.01$, Partial $\mathrm{Eta}^{2}=.31$; there was also a significant three-way interaction between schedule, trial, and instruction group, $F(9,93)=1.95, p=.05$, Partial $\mathrm{Eta}^{2}=.159$. There was a marginal interaction between schedule and instruction group, $F(3,31)=2.36, p=.09$, Partial $\mathrm{Eta}^{2}=$ .186. There were no other statistically significant main effects or interactions, all $p \mathrm{~s}>.10$.

To further explore these data, a two-way ANOVA (schedule $\mathrm{x}$ trial) was conducted for each separate instruction group. For the Minimal instructions group a main statistical effect of schedule was shown, $F(1,7)=5.64, p<.05$, no main effect was seen for trial, $p>.10$, and a marginal interaction was shown between schedule and trial, $F(3,21)=2.82, p=.064$.

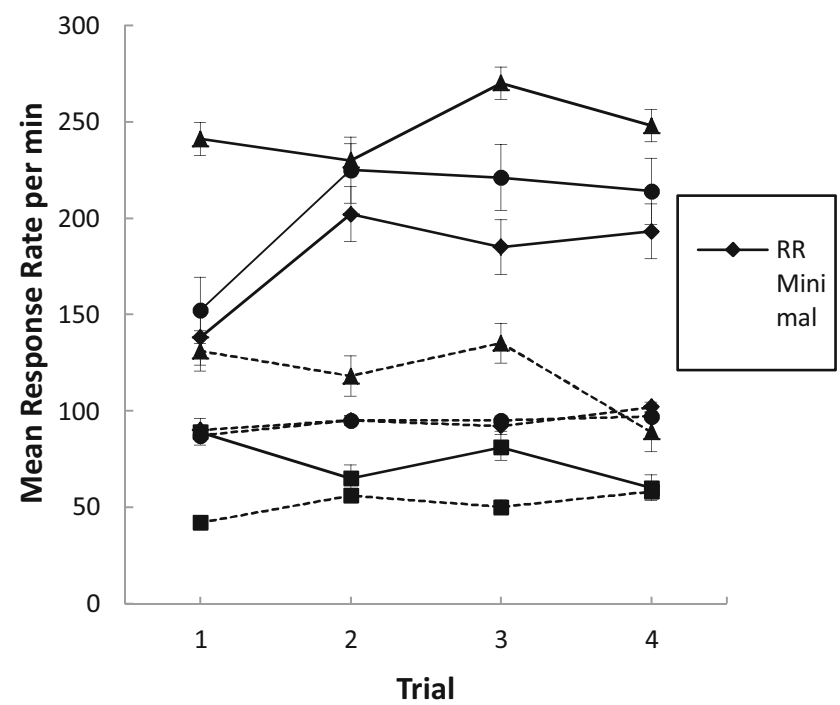

Fig. 1 Experiment 2, mean response rates per minute - RR and RI trials for each instruction group

For the Go Fast group, a statistically significant main effect of schedule was shown, $F(1,8)=12.92, p<.01$, but no statistically significant effects for trial, or for the interaction, $p \mathrm{~s}>.10$. No statistically significant effects were shown for the Go Slow group, all $p \mathrm{~s}>.10$. For the Accurate Rate instruction group, a statistically significant main effect was shown for schedule, $F(1,7)=8.29, p<.05$, but no other statistically significant effects were found, all $p s>0.1$.

The results of these analyses indicate that, overall, participants responded in a differentiated manner according to schedule type, and according to instruction group, with participants in the Minimal, Go Fast, and Accurate Rate groups showing differentiated and sensitive schedule performance. These results are somewhat consistent with those of Hayes et al. (1986a), who found that, while verbally instructed participants performed in a more differentiated and schedule-sensitive manner than noninstructed participants, some noninstructed participants also showed similar schedule-sensitive differentiation. The results are also reminiscent of those of Catania and colleagues (Catania et al., 1982; Shimoff et al., 1981), who reported inconsistent relationships between verbal instructions and both RR-RI performance differences and sensitive responding to RR-RI contingencies.

\section{Performance awareness}

Summed performance awareness (PA) scores for every trial ranged from 0 to 16 (in multiples of 2) across the sample, with a mean of $6.46( \pm 4.39)$. The sample was split into two groups according to a median split of performance awareness scores. The median PA score was 6; consequently, scores between 0 and 6 were considered to have relatively lower performance awareness, while scores between 8 and 16 were described as 
showing higher performance awareness. For this experiment, Group Lower consisted of 22 participants (mean PA score = $3.64 \pm 2.01$ ), whilst Group Higher consisted of 13 participants (mean PA score $=11.23 \pm 2.89$ ).

Figure 2 presents the response rates for each exposure to each of the two schedule types (RR and RI), for Higher PA and Lower PA groups. A three-factor, mixed-model ANOVA (PA Group $x$ schedule $x$ trial) was conducted on these data and showed a statistically significant main effect of schedule, $F(1$, $33)=56.19, p<.001$, Partial $\mathrm{Eta}^{2}=.63$, but no statistically significant effect of trial, $p>0.1$ or of PA group, $F<1$. There was no statistically significant finding for the interaction between trial and PA group, $F<1$; however, there were statistically significant findings for the interactions between schedule and PA group, $F(1,33)=23.17, p<.001$, Partial $\mathrm{Eta}^{2}=.413$, schedule and trial, $F(3,99)=4.32, p<.01$, and schedule, trial, and PA group, $F(3,99)=9.44, p<.001$, Partial $\mathrm{Eta}^{2}=.222$.

To further investigate these findings, two-factor AVOVAs (schedule $\mathrm{x}$ trial) were conducted on the data for higher and lower PA groups. For Group Lower PA, a statistically significant main effect was found for schedule, $F(1,21)=7.22, p<$ .05 , Partial $\mathrm{Eta}^{2}=.26$, but no other effects were found, all $p \mathrm{~s}>$ .10. For Group Higher PA, a statistically significant main effect of schedule was found, $F(1,12)=38.21, p<.001$, Partial $\mathrm{Eta}^{2}=.76$, and a two-way interaction between schedule and trial was shown, $F(3,36)=11.51, p<.001$, Partial $\operatorname{Eta}^{2}=.49$. No statistically significant effect was found for trial, $p>.10$.

Taken together, these results indicate that performance awareness was associated with response rates according to schedule type, with slightly larger RR-RI response differences apparent for higher PA than for lower PA participants; although both PA groups showed differentiated schedule responding sensitive with the contingencies, the effect sizes indicate that PA was more strongly related with RR-RI performance differentiation than was instruction group. These results are consistent with those of Catania et al. (1982), who found that while shaping responses versus verbally

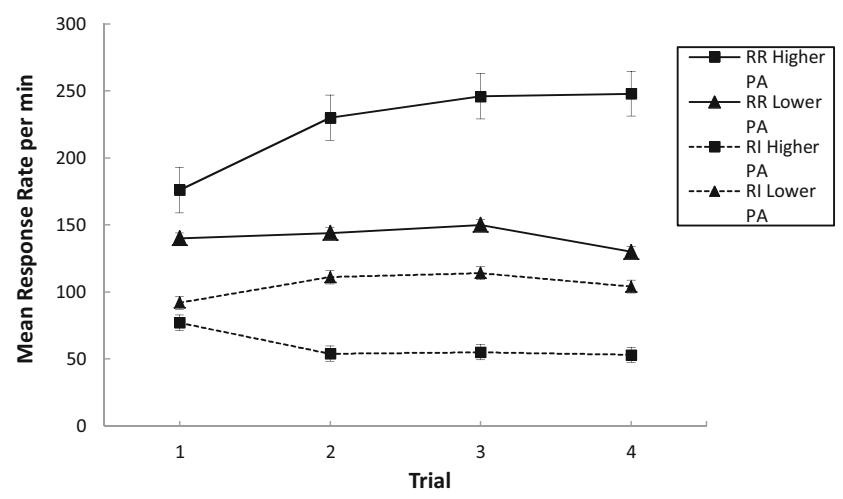

Fig. 2 Experiment 1, mean response rates per minute - RR and RI trials for higher and lower performance awareness instructing participants produced inconsistent sensitivity to the RR-RI contingencies, RR-RI response differences among the groups were consistent with correct guesses about the contingencies. They are also consistent with findings by Wearden and Shimp (1985), which suggested that schedulesensitive responding was reliably related to PA in RI schedules. Thus, for Experiment 1, performance awareness was consistently related to schedule-sensitive responding, while verbally instructed responding was not.

\section{Association between instruction group and PA}

To further investigate the relationship between performance awareness and instruction group, the categorical data defining these two factors were entered into a Pearson's hi-square analysis of association. Of the participants who had received minimal instructions, three were classified as having lower PA while five had higher PA. Of those who received ratioschedule appropriate (Go Fast) instructions, five had lower PA whereas four had higher PA. Of those who received interval-schedule (Go Slow) instructions, 10 had lower PA and zero had higher PA. Of those who received accurate-rate instructions, four had lower PA and four had higher PA. Chisquare analysis indicated $\mathrm{X}^{2}(3, N=35)=8.88, p=0.03$. The cross tabulation suggests that the reason for this significant association is the absence of any high-performance-aware participants in the Go Slow group. In other instruction groups, the participants were split almost equally between low PA and high PA, with the Minimal instructions group comprising a greater number of higher PA participants than the Accurate Rate group. Thus, it can be observed from these data that providing participants with accurate instructions regarding the most efficient manner of point scoring was no more likely to result in accurate guesses about sensitive performance than providing no accurate instructions.

The results reported here for Experiment 1 resemble those reported by Hayes et al. (1986a) in terms of the degree to which the participants appeared to understand the schedule contingencies. Hayes et al. (1986a) reported that of the minimally instructed participants, six showed efficient differential responding while four did not; in the current study, of the minimally instructed participants, five showed higher PA whilst three showed lower PA. The split between Go Fast participants, reported by Hayes et al. (1986a), indicated five participants showing differential responding, whilst two did not; the current results showed four Go Fast participants with higher PA and five with lower PA. Hayes et al. (1986a) reported one participant to show differential responding in the Go Slow group, with nine showing nondifferential responding; current results indicated that no participants in the Go Slow group showed higher PA whilst 10 showed lower PA. Only the results of the Accurate rate group differed somewhat between the two studies. The current 
results show four of these participants demonstrating higher PA and four demonstrating lower PA, whereas in the results reported by Hayes et al. (1986a), 15 out of 16 participants showed efficient differential responding. These mostly consistent results between the two studies suggest that minimal instructions, or those pertaining to RR schedules only, are not likely to eliminate the likelihood of sensitive schedule responding or of accurately describing the contingency but that RI-appropriate instructions are likely to decrease schedule-sensitive responding and accurate description of the contingency, and that accurate instructions may increase the likelihood of schedule-sensitive responding but not accurate description of the performance required to respond sensitively to the schedules. This might be due to the fact that RIappropriate instructions reduce variability of responses and thus the chance of making contact with an RR contingency, as suggested by Cerutti (1989), Galizio (1979), and Joyce and Chase (1990).

\section{Experiment 2}

The results of Experiment 1, suggest that although both verbal instructions provided by the experimenter and performance awareness (PA) can relate to RR-RI schedule performance, PA was more strongly related to schedule-sensitive responding and not related to verbal instructions. It might have been intuitively predicted that participants given accurate instructions concerning how to score points would show greater performance awareness to the schedules than those who had received no such instructions, but this was not observed in the findings from Experiment 1. To further explore these factors, a second experiment aimed to consider the extent to which participants would continue to show schedule-sensitive responding in relation to both experimenter-provided instructions and PA after the removal of reinforcement. Although several studies have examined this issue (Hayes et al., 1986a; Hayes et al., 1986b; Shimoff et al., 1981), this effect has not been examined using the relatively simple schedule employed in Experiment 1. To this end, after initial conditioning on a multiple RR-RI schedule, reinforcement was withdrawn after eight trials to investigate any differences that may occur in extinction between participants who were verbally instructed and those who were not. Extinction tests have been used previously in the examination of the impact of instructions on responding, where it has been found that instructed performance (as opposed to contingency shaped) is more resistant to extinction (see Hayes et al., 1986a). The second experiment aimed to investigate the performance of participants during a period of extinction in relation to both verbal instructions provided by the experimenter and to performance awareness.

\section{Method}

\section{Participants}

A sample of 33 students was recruited via the Psychology Department subject-pool system; they received credit for their participation, but no financial payment. The sample comprised 21 females and 12 males, aged between 19 and 50 years (mean $=22.94 \pm 6.5$ ).

\section{Apparatus}

The same alternating schedule of yoked RR and RI trials was used as in Experiment 1,; however, the task comprised 20 trials, which were each 2 minutes in length. Reinforcement was delivered in the first eight conditioning trials (four RR and four RI, respectively) according to an RR-20 schedule. The remaining six trials were extinction trials, whereby no reinforcement was delivered at all. Participants were not informed that no points could be earned after the first eight trials. The color of the stimulus box was counterbalanced for both conditioning trials and for extinction trials so that for half the sample, the rectangle in the upper center of the screen was red for the RR trials and green for RI trials. For the other half of the sample, the rectangle was green for the RR trial and red for the RI trial. For half the sample who had viewed a red rectangle for RR trials and green rectangle for RI trials, the first extinction trial showed a red stimulus box, followed by green, alternating for the remainder of the trials. For the other half, the rectangle in the first extinction trial was green, followed by red for the second, recurring. This counterbalancing also occurred for the remainder of the sample who had viewed a green color stimulus box for the first (RR) conditioning trial and a red box for the second (RI) trial.

\section{Procedure}

The procedure was identical to that of Experiment 1, except for the instructions given to the participants. Participants were placed into one of two experimental conditions, Minimal instructions and Accurate Rate instructions. All participants received identical initial instructions to those given in Experiment 1, except that they were informed that there would be 20 trials. Following these initial on-screen instructions, participants viewed a further screen before the task began. Those in the Minimal instructions group received the same instructions as the equivalent group in Experiment 1,:

Remember, your task is to score as many points as possible by pushing the space bar. 
Those in the Accurate Rate instructions group received the same instructions as in the equivalent group in Experiment 1, (after Hayes et al., 1986a):

Remember, your task is to score as many points as possible by pushing the space bar. The rectangle which changes color on the trials is important. When it is one color, rapid pushes on the space bar will work best. When it is the other color, pushes with several seconds in between them will work best.

The Minimal instructions group consisted of 16 participants, and the Accurate Rate instructions group consisted of 17 participants. Participants were tested individually in a quiet room. After completion of the task, participants completed a performance-awareness questionnaire, as used in Experiment 1 , The same question was posed for each of the 20 trials, with the response options identical to the one previously used; however, performance-awareness scores were only calculated using responses to the first eight conditioning trials, as it was not possible to show awareness of how to score points in extinction trials since no points were available.

\section{Results and discussion}

\section{Experimenter instructions}

Figure 3 presents the response rates for each exposure to each of the two schedule types (RR and RI) for the two groups of participants (Minimal instructions and Accurate Rate instructions) for the conditioning trials (trials 1-4). A three-factor, mixed-model ANOVA (instruction group $\mathrm{x}$ schedule $\mathrm{x}$ trial) was conducted on these data and showed a statistically significant main effect of schedule, $F(1,31)=13.54, p<.001$, Partial $\mathrm{Eta}^{2}=.304$, but no statistically significant main effects of trial or instruction group, $F_{\mathrm{S}}<1$. There were no statistically significant interactions for these data, all $p s>.10$. Thus, participants receiving accurate instructions were no more likely

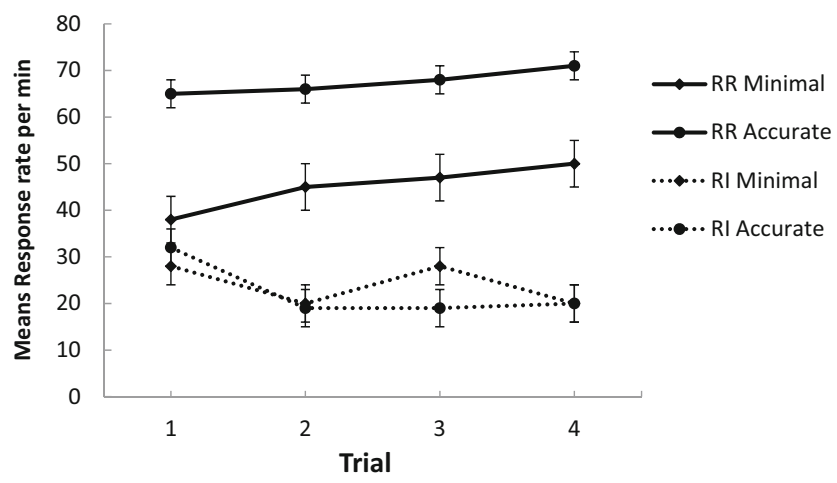

Fig. 3 Experiment 2 mean response rates per minute - RR and RI conditioning trials (1-4) for each instruction group to show differentiated RR-RI responses than those receiving minimal instructions, consistent with findings by Catania et al. (1982) and Shimoff et al. (1981), which indicated that verbal instructions did not produce consistent RR-RI response-rate differences or schedule-sensitive responding.

Figure 4 presents the response rates for each exposure to each of the two schedule types (RR and RI) for the two groups of participants (Minimal instructions and Accurate Rate instructions) for the three extinction trials for each schedule. A three-factor, mixed-model ANOVA (instruction group x schedule $x$ trial) was conducted on these data for these factors for the extinction trials, and showed no statistically significant main effects for schedule or for instruction group, $F_{\mathrm{S}}<1$. There was a statistically significant main effect for trial, $F(2,60)=4.01, p$ $<.05$, Partial $\mathrm{Eta}^{2}=.118$. There were no statistically significant interactions between any factors, all $p s>.20$.

In an attempt to accommodate the differences in response rate seen in training, the mean rates of responding during the three extinction trials were expressed as a proportion of the mean rates of responding seen over the four training trials. This produced a mean proportion of baseline responding for the minimal instruction group of $\mathrm{RR}=.84( \pm .63)$ and $\mathrm{RI}=1.10$ ( \pm .33$)$; and for the accurate instruction group these data were $\mathrm{RR}=.55( \pm .43)$ and $\mathrm{RI}=1.78( \pm 3.11)$. A two-factor ANOVA (group $\mathrm{x}$ schedule) conducted on these revealed a marginally significant main effect of schedule, $F(1,31)=2.99, .09>p>$ .08 , Partial $\mathrm{Eta}^{2}=.088$, but no significant main effect of group, $p>.20$, nor interaction between the factors, $F<1$.

These findings show that extinction occurred, and that there was a tendency for the response rate in extinction to decrease more, relative to baseline, in the RR component than in the RI component. However, there was little effect of instruction type on extinction. This latter finding is inconsistent with those of Joyce and Chase (1990), who found that accurately instructed participants showed little sensitivity to a change in the schedule contingency, and those of Hayes et al. (1986a) who found that accurately-instructed participants

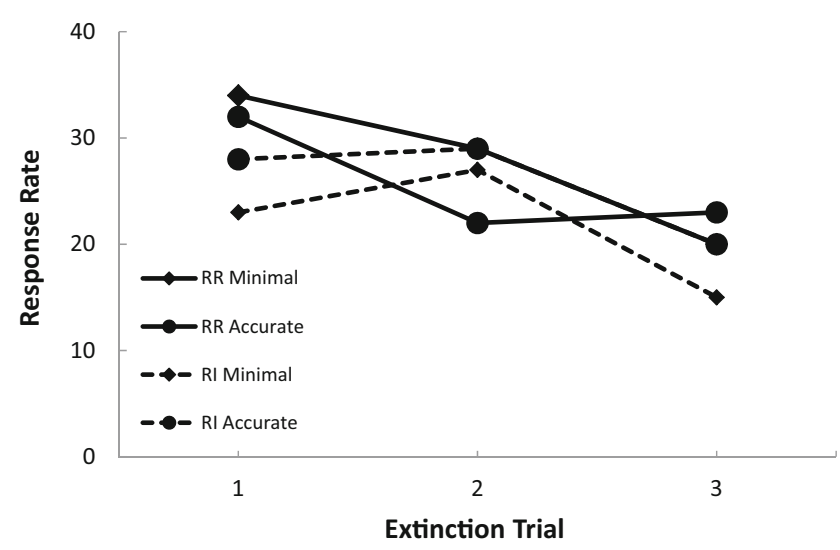

Fig. 4 Experiment 1, mean response rates per minute - RR and RI extinction trials (5-10) for each instruction group. 
showed few extinction effects. The reason for this inconsistency is unclear, but may relate to the use of a simpler contingency in the current study

\section{Performance awareness}

Summed performance awareness scores across the trials ranged from 0 to 16 , with a mean of $6.73( \pm 4.24)$ and a median of 6 . The sample was split into two groups according to their performance awareness score as described in Experiment 1; a score of 0 to 6 was classed as Lower PA, while a score of 8 to 16 was classed as Higher PA. Group Lower consisted of 18 participants (mean PA score $=3.56 \pm 2.23$ ) and Group Higher consisted of 15 participants (mean PA score $=10.53 \pm 2.56$ ).

Figure 5 presents the response rates for each exposure to each of the two schedule types (RR and RI), for Higher PA and Lower PA groups for the conditioning trials (trials 1-4). A three-factor, mixed-model ANOVA (PA Group x schedule $\mathrm{x}$ trial) was conducted on the data for the conditioning trials (trials 1-4) and showed a statistically significant main effect of schedule, $F(1,31)=20.46 p<.001$, Partial $\mathrm{Eta}^{2}=.398$, and of PA Group, $F(1,31)=4.42, p=.044$, Partial $\mathrm{Eta}^{2}=.125$; but no statistically significant effect of trial, $F>1$. There was no statistically significant finding for the interaction between trial and PA group, $F>1$. There was a statistically significant finding for the interaction between schedule and PA group, $F(1,31)=11.26, p=.002$, Partial $\mathrm{Eta}^{2}=.266$. However, there were no statistically significant findings for the interactions between schedule and trial, $F(3,93)=$ $2.42, p=.07$, or for schedule, trial, and PA group, $p>0.1$. The effect sizes suggest that performance awareness was a stronger mediator of schedule-sensitive responding than instruction group, in accordance with findings by Catania et al. (1982), and with the findings of the current Experiment 1,.

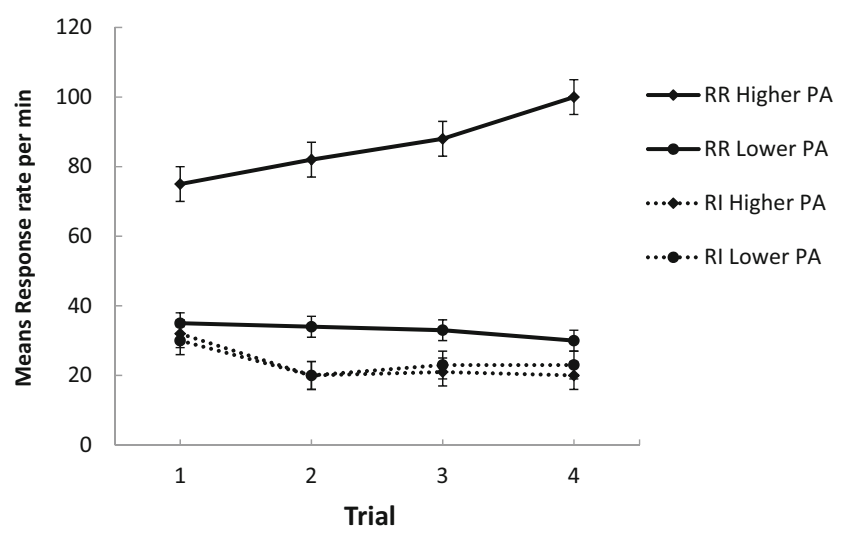

Fig. 5 Experiment 2 mean response rates per minute - RR and RI conditioning trials $(1-4)$ for higher and lower performance awareness
Figure 6 presents the response rates for each exposure to each of the two schedule types (RR and RI), for Higher PA and Lower PA groups for the extinction trials. A three-factor, mixed-model ANOVA was conducted using the same factors for the extinction trials (trials 5-10) and showed no statistically significant main effects for schedule or for PA group, $p>$ .10 , for both factors. There was a statistically significant main effect of trial, $F(2,62)=6.52, p<.001$, Partial $\mathrm{Eta}^{2}=.174$. There were no statistically significant interactions between schedule and PA group, $p>.40$, schedule and trial, $p>.10$, or schedule, trial and group, $F<1$. The interaction between trial and PA group was marginally significant, $F(2,62)=2.87$, $p=.064$, Partial $\mathrm{Eta}^{2}=.085$. Mean scores for both groups indicate that there was a slight tendency for those with higher PA to extinguish faster than those with lower PA. Effect sizes indicate no substantial differences between results for instruction group and results for PA group in this respect.

The mean rates of responding during the three extinction trials, expressed as a proportion of the mean rates of responding seen over the four training trials, for the lower PA group were $\mathrm{RR}=.97( \pm .65)$ and $\mathrm{RI}=1.03$ $( \pm .34)$; and for the higher PA group these data were $\mathrm{RR}=.46( \pm .30)$ and $\mathrm{RI}=1.80( \pm 3.09)$. Although there appeared to be a greater extinction effect for the RR schedule, a two-factor ANOVA (group x schedule) conducted on these revealed no statistically significant main effects or interaction, all $p \mathrm{~s}>.10$.

These data do not provide any clear support for the findings reported by Hayes et al. (1986a), who suggested that extinction occurred more rapidly in participants who had previously performed sensitively to the contingency under conditioning trials, but whom could not have done so as a result of experimenter instructions. Thus, the findings of Hayes et al. (1986a) seem to suggest that a degree of performance awareness is more important than instructions received in extinguishing rapidly when reinforcement is withdrawn. Our results show no such distinction.

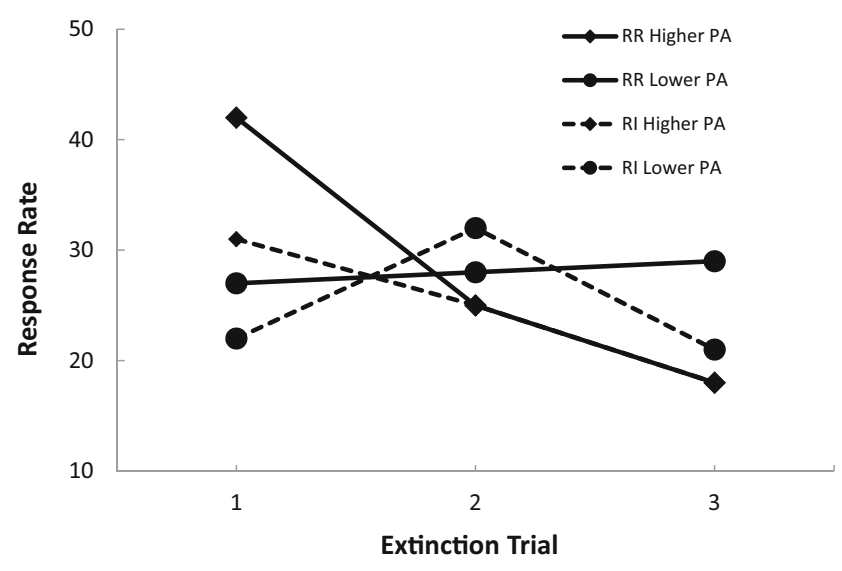

Fig. 6 Experiment 2 mean response rates per minute - RR and RI extinction trials (5-10) for higher and lower performance awareness 


\section{Association between instruction group and PA}

A Pearson's chi-square analysis of association was conducted on the data to further investigate the relationship between performance awareness and instruction group. It was found that of the 16 participants who received minimal instructions, nine had lower performance awareness whilst seven had higher PA. Of the 17 who received accurate instructions, nine had lower PA whilst seven had higher PA; $\mathrm{X}^{2}(1, N=33)=$ $.36, p>.8$. Thus, there was no relationship between instruction group and performance awareness in Experiment 2, consistent with the results of Experiment 1,

Hayes et al. (1986a) use the term "apparent schedule sensitivity" to describe the differential responding by participants in their study, and make the point that humans may respond in a way which appears schedule sensitive but which is not controlled by the schedules. They suggest that only behavior which is sensitive to changes in the contingencies can truly be described as schedule sensitive. The current study attempted to measure whether participants are responding sensitively to schedule contingencies, as in previous studies. By measuring performance awareness, we found that PA was reliably associated with apparent schedule sensitivity (see Bradshaw \& Reed, 2012). Of course, being able to accurately describe how to score points does not guarantee that other factors could not be enabling schedule-sensitive responding, but it does seem to suggest that participants are performing in a truly schedule-sensitive manner. Furthermore, our results in this study also suggest that this performance awareness is independent of verbal instructions provided by experimenters. However, the results from the extinction trials in Experiment 2 of the present study are unclear. While higher PA participants showed more rapid extinction effects that lower PA participants, it does not appear that higher performance awareness produced stronger extinction effects than accurate instructions, which might have been expected after consideration of the results by Hayes et al. (1986a). Our results are consistent with findings by Catania et al. (1982), who reported that neither shaped nor instructed responses produced consistent differences in RR and RI responding, but a relationship could be observed between schedule-sensitive responding and higher PA; whereas, if participants had been instructed, the relationship between response rates and PA was much more inconsistent.

\section{Experiment 3}

The results of Experiments 1, and 2 suggest that performance awareness is more strongly related to schedule-sensitive responding than to the instructions provided by the experimenter and that PA and instruction group showed no relationship with one another; that is, that participants provided with accurate-rate instructions were no more likely to show higher PA than those who received minimal instructions. Measurement of performance awareness for both of the first two experiments was conducted through retrospective, closedended questionnaires. Measuring PA retrospectively was deemed necessary to avoid any interference effects which might be observed if participants were asked about their performance and provided with options as to how best to increase points after each trial or RR-RI trial pair, rather than posttask. However, for both experiments, participants were required to recall their opinions of the best way to increase their points score by thinking back over several trials, and in the case of the second experiment, they needed to recall 20 separate trials in order to attempt this. Despite the reliable association between RR and RI differential responding and PA shown in these experiments, which are also consistent with findings from our previous study (Bradshaw \& Reed, 2012), it is not inconceivable that participants struggled to recall the best way to increase their points in each trial and were using the fixedchoice options as prompts. In order to address this, a third experiment was conducted that was functionally identical to Experiment 2 but aimed to vary the measurement of PA between groups to test reliability of the closed-ended PA measure.

\section{Method}

\section{Participants}

A sample of 32 students was recruited via the Psychology Department subject-pool system; they received credit for their participation, but no financial payment. The sample comprised 29 females and 3 males, aged between 18 and 26 years $($ mean $=19.59 \pm 1.64)$.

\section{Apparatus}

The same alternating schedule of yoked RR and RI trials was used as in Experiment 2; the task comprised 14 trials, each $2 \mathrm{~min}$ in length. Reinforcement was delivered in the first eight conditioning trials (four RR and four RI, respectively) according to an RR-20 schedule. The remaining six trials were extinction trials, whereby no reinforcement was delivered at all. Participants were not informed that no points could be earned after the first eight trials. The color of the stimulus box was counterbalanced for both conditioning trials and for extinction trials, so that, for half the sample, the rectangle in the upper center of the screen was red for the RR trials and green for RI trials. For the other half of the sample, the rectangle was green for the RR trial and red for the RI trial. For half the sample who had viewed a red rectangle for RR trials and green rectangle for RI trials, the first extinction trial showed a red stimulus box, followed by green, alternating for the remainder of 
the trials. For the other half, the rectangle in the first extinction trial was green, followed by red for the second, recurring. This counterbalancing also occurred for the remainder of the sample who had viewed a green color stimulus box for the first (RR) conditioning trial and a red box for the second (RI) trial.

\section{Procedure}

Participants were split into four experimental groups. As in Experiment 2, half of the participants received Minimal instructions and the other half received Accurate Rate instructions. The instructions given to each of these groups were identical to those used in Experiment 2. In addition to this, half of the participants in each of these groups received closed-ended posttask performance-awareness questions identical to those used in Experiment 2, while the other half received openended posttask performance-awareness questions.

The closed-ended questionnaire was identical to that described in Experiment 1, and was scored in the same way, using only the conditioning trial responses as described in Experiment 2. The open-ended questions were posed for each of the 14 trials completed in the task and used identical wording to the closed-ended questions ("In the [first/second] game in the task, what did you consider to be the best way to increase your points score?"). Space was provided immediately following each of these questions for participants to respond in their own words.

Content analysis was performed by two independent raters on these responses to score performance awareness, consistent with procedures used by Bradshaw and Reed (2012); Osborne and Reed (2008); and Vaughn, Schumm, and Sinagub (1996). Two points were awarded for responses which accurately described the contingency, one point was awarded for responses thought to partially describe the preceding contingency, and zero points were awarded for an inappropriate, inaccurate, or uncertain response (see Bradshaw \& Reed, 2012; Catania et al., 1982; Matthews et al., 1985; Shimoff et al., 1986). A Cohen's kappa analysis was used to check the interrater reliability of these scores. A high mean reliability of 0.85 was identified between their separate judgments of the participants' responses.

There were eight participants in each of the experimental groups. Participants were tested individually in a quiet room and completed the performance awareness questionnaire (open or closed) immediately after completing the task.

\section{Results and discussion}

\section{Experimenter instructions}

Figure 7 presents the response rates for each exposure to each of the two schedule types (RR and RI), for the two groups of participants split by instruction type (Minimal instructions and

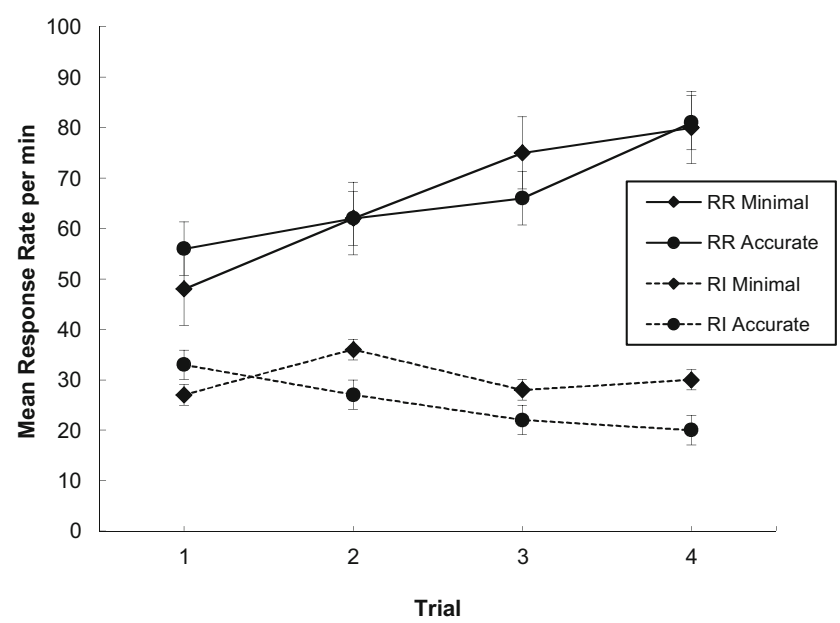

Fig. 7 Experiment 3 mean response rates per minute - RR and RI conditioning trials $(1-4)$ for each instruction group

Accurate Rate instructions), for the conditioning trials (trials 14). A three-factor, mixed-model ANOVA (instruction group $x$ schedule $\mathrm{x}$ trial) was conducted on these data, and, consistent with Experiment 2, showed a statistically significant main effect of schedule, $F(1,30)=20.35, p<.001$, Partial $\mathrm{Eta}^{2}=.404$, but no statistically significant main effects of trial, $p>.05$ or instruction group, $F<1$. There were no statistically significant interactions for these data, all $F_{\mathbf{s}}<1$. Thus, as with Experiment 2 , participants receiving accurate instructions were no more likely to show differentiated RR-RI responses than those receiving minimal instructions, corroborating findings by Catania et al. (1982) and Shimoff et al. (1981), which indicated that verbal instructions did not produce consistent RR-RI response-rate differences or schedule-sensitive responding.

Figure 8 presents the response rates for each exposure to each of the two schedule types (RR and RI), for participants split by instruction type (Minimal instructions and Accurate Rate instructions), for the extinction trials. A three-factor, mixed-model ANOVA (instruction group $\mathrm{x}$ schedule $\mathrm{x}$ trial) was conducted on these data for these factors for the extinction trials, and showed only a statistically significant main effect of

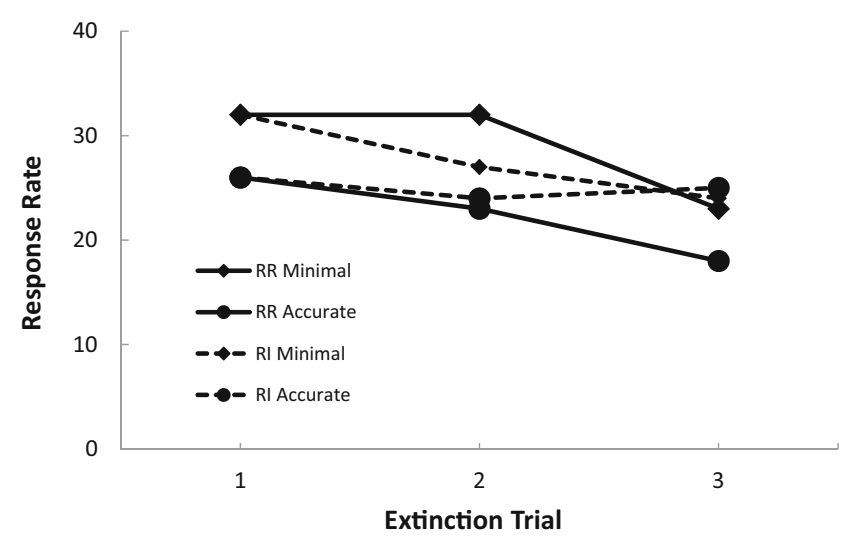

Fig. 8 Experiment 3 mean response rates per minute - RR and RI extinction trials (5-10) for each instruction group 
trial, $F(2,60)=4.01, p<.05$, Partial $\mathrm{Eta}^{2}=.118$. There were no other statistically significant main effects or interactions, all $p \mathrm{~s}>.20$. The mean rates of responding during the three extinction trials, expressed as a proportion of the mean rates of responding seen over the four training trials, produced a mean proportion of baseline responding for the minimal instruction group of $\mathrm{RR}=.62( \pm .53)$ and $\mathrm{RI}=1.13( \pm .59)$; and for the accurate instruction group these data were $\mathrm{RR}=.63( \pm .52)$ and $\mathrm{RI}=.99( \pm .59)$. A two-factor ANOVA (group x schedule) conducted on these revealed a significant main effect of schedule, $F(1,30)=12.36, p<.001$, Partial $\mathrm{Eta}^{2}=.292$, but no significant main effect of group nor interaction between the factors, both $F \mathrm{~s}<1$. Thus, although there was an effect of extinction, and this was greater for the RR than for the RI schedule, the Accurate Rate instruction group was no less likely to show extinction than the Minimal instructions group, consistent with the findings of Experiment 2.

\section{Performance awareness for the whole sample}

Summed performance awareness scores across the trials for the whole sample ranged from 0 to 16 , with a mean of 7.25 $( \pm 5.88)$. The sample was split into two groups according to their performance awareness score as described in Experiment 1; a score of 0 to 6 was classed as Lower PA while a score 7 to 16 was classed as Higher PA. Group Lower consisted of 16 participants (mean PA score $=2.25 \pm 2.51$ ) and Group Higher consisted of 16 participants (mean PA score $=12.25 \pm 3.42$ ).

Figure 9 presents the response rates for each exposure to each of the two schedule types (RR and RI), for Higher PA and Lower PA groups for the conditioning trials (trials 1-4) for the whole sample. A three-factor, mixed-model ANOVA (PA Group $\mathrm{x}$ schedule $\mathrm{x}$ trial) was conducted on the data for the conditioning trials (trials 1-4) and showed a statistically significant main effect of schedule, $F(1,30)=21.19 p<.001$,

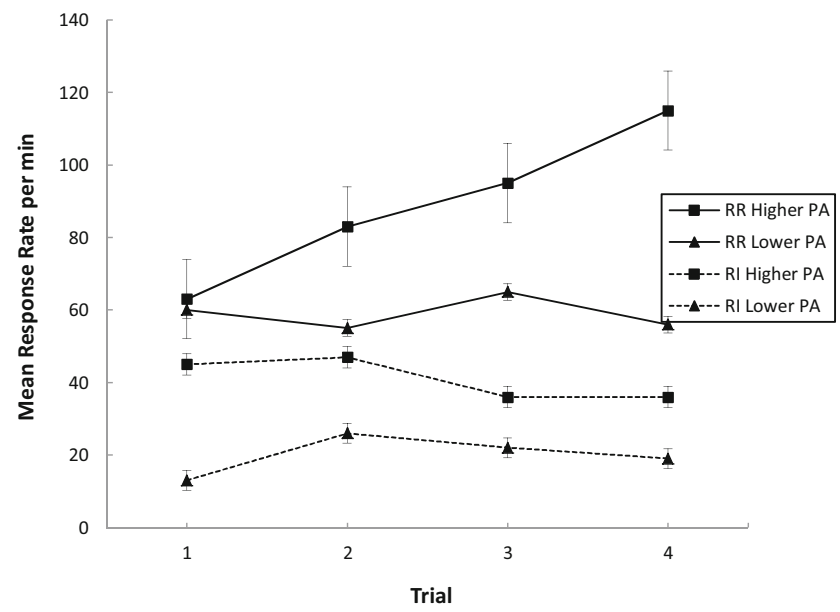

Fig. 9 Experiment 3 mean response rates per minute - RR and RI conditioning trials (1-4) for higher and lower performance awareness
Partial $\operatorname{Eta}^{2}=.414$, and of PA Group, $F(1,30)=8.10, p<.01$; but no statistically significant effect of trial, $p>.1$. There was no statistically significant interactions between trial and PA group, $p>.2$ or schedule and PA group, $p>.2$. However, there were statistically significant findings for the interactions between schedule and trial, $F(3,90)=7.86, p<.001$ and for schedule, trial, and PA group, $F(3,90)=5.05, p=.003$. These findings, in comparison to the nonsignificant interactions in the experimenter instructions data, suggest that performance awareness was a stronger mediator of schedule-sensitive responding than instruction group, in accordance with the findings of Experiments 1, and 2 and findings by Catania et al. (1982).

Figure 10 presents the response rates for each exposure to each of the two schedule types (RR and RI), for Higher PA and Lower PA groups for the extinction trials for the whole sample. A three-factor, mixed-model ANOVA (schedule $\mathrm{x}$ trial $\mathrm{x}$ group) revealed a statistically significant main effect of trial, $F(2,60)=4 . .38, p<.05$, Partial $\mathrm{Eta}^{2}=.127$, and a significant interaction between group and trial, $F(2,60)=$ $3.20, p<.05$, Partial $\mathrm{Eta}^{2}=.096$. No other main effects or interactions were significant, all $p \mathrm{~s}>.20$. The mean rates of responding during the three extinction trials, expressed as a proportion of the mean rates of responding seen over the four training trials, produced a mean proportion of baseline responding for the lower PA group: $\mathrm{RR}=.79( \pm .64)$ and $\mathrm{RI}$ $=1.03( \pm .52)$; and for the higher PA group these data were RR $=.46( \pm .30)$ and $\mathrm{RI}=1.09( \pm .66)$. A two-factor ANOVA (group $\mathrm{x}$ schedule) conducted on these revealed a significant main effect of schedule, $F(1,30)=13.17, p<.001$, Partial $\mathrm{Eta}^{2}=.305$, but no significant main effect of group, nor interaction between the factors, both $p \mathrm{~s}>.10$. Thus, findings indicate that neither higher nor lower PA groups showed stronger extinction effects.

\section{Open-ended PA questionnaire}

Figure 11 displays the response rates for each exposure to each of the two schedule types (RR and RI), for Higher PA and Lower PA groups, for the conditioning trials (trials 1-4), for the open-ended PA questionnaire group. A three-factor, mixed-model ANOVA was conducted using the same factors for the conditioning trials (trials 1-4) for this group, and showed statistically significant main effects for schedule, $F(1,14)=5.64, p<.05$, Partial $\mathrm{Eta}^{2}=.287$ and for PA group, $F(1,14)=12.71, p=.003$, Partial $\mathrm{Eta}^{2}=.476$. There was no statistically significant main effect for trial, $p>.1$. There was a statistically significant interaction for schedule $\mathrm{x}$ PA group, $F(1,14)=5.75, p<.05$, Partial $\mathrm{Eta}^{2}=.291$. There were no other statistically significant interactions, $p \mathrm{~s}>.10$.

Figure 12 presents the response rates for each exposure to each of the two schedule types (RR and RI) for Higher PA and Lower PA groups for the extinction trials for the open-ended 


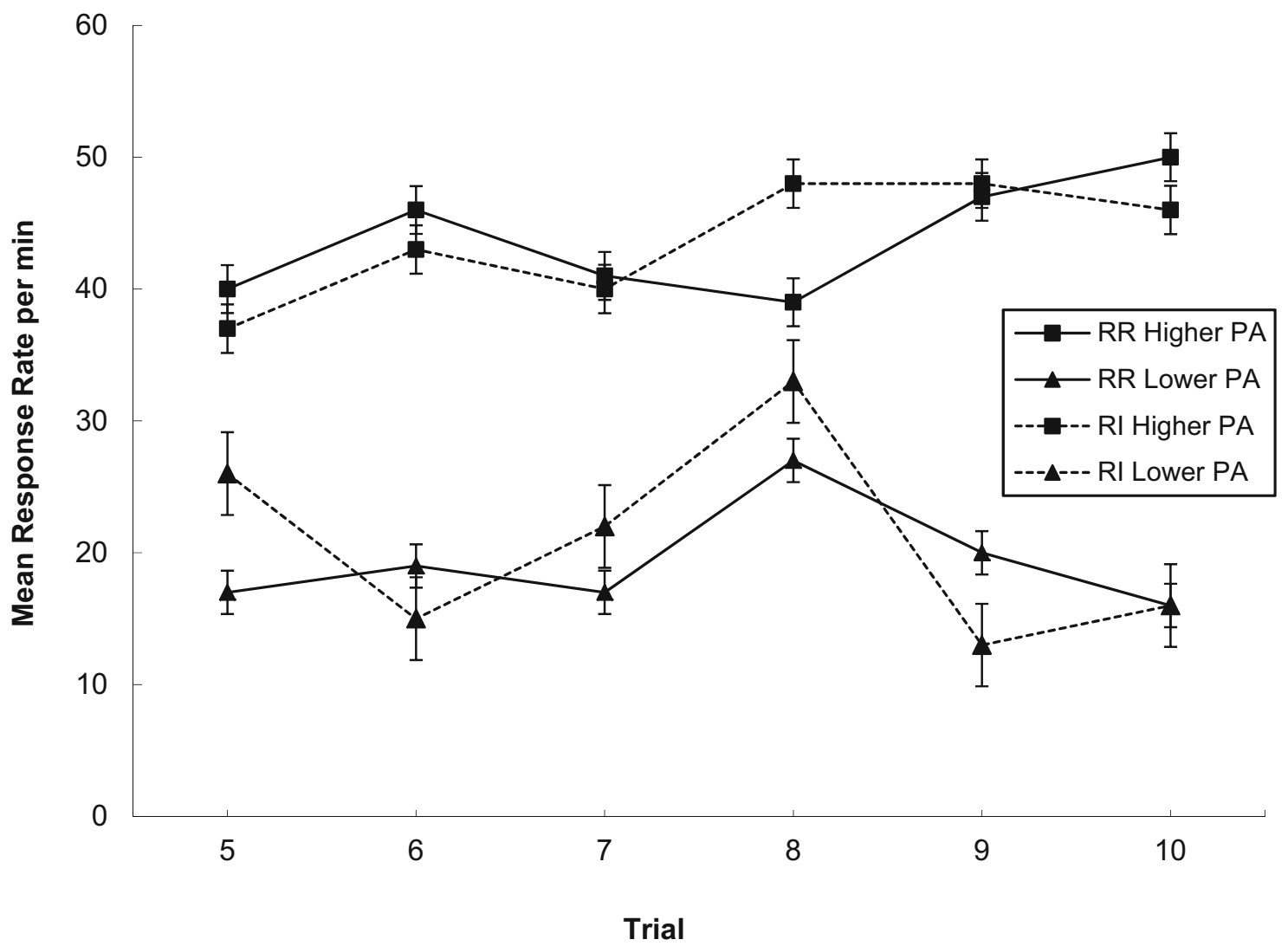

Fig. 10 Experiment 3 mean response rates per minute - RR and RI extinction trials (5-10) for higher and lower performance awareness

PA questionnaire group. A three-factor, mixed-model ANOVA (schedule $\mathrm{x}$ trial $\mathrm{x}$ PA group) showed a marginally significant main effect for trial, $F(2,28)=2.79, .08>p>.07$, Partial $\mathrm{Eta}^{2}=.135$, and for the interaction between PA group and trial, $F(2,28)=2.93, .07>p>.06$, Partial $\mathrm{Eta}^{2}=.174$. There were no other statistically significant main effects or interactions, all $p \mathrm{~s}>.1$.

The mean rates of responding during the three extinction trials, expressed as a proportion of the mean rates of

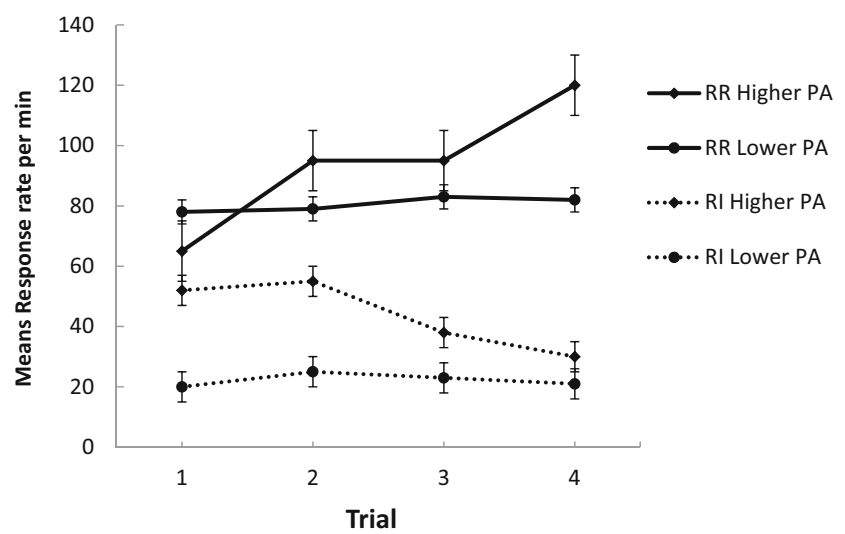

Fig. 11 Experiment 3 mean response rates per minute - RR and RI conditioning trials (1-4) for higher and lower performance awareness using open-ended PA measure responding seen over the four training trials, produced a mean proportion of baseline responding for the lower PA group: RR $=1.07( \pm .75)$ and $\mathrm{RI}=1.07( \pm .53)$; and for the higher PA group these data were $\mathrm{RR}=.58( \pm .31)$ and $\mathrm{RI}=1.16( \pm .68)$. A two-factor ANOVA (group x schedule) conducted on these revealed a marginally significant main effect of schedule, $F(1$, $14)=3.91, .07>p>.06$, Partial $\mathrm{Eta}^{2}=.218$, and a statistically significant schedule $\mathrm{x}$ group interaction, $F(1,14)=4.21, p<$ .05 , Partial $\mathrm{Eta}^{2}=.231$, but no significant main effect of group,

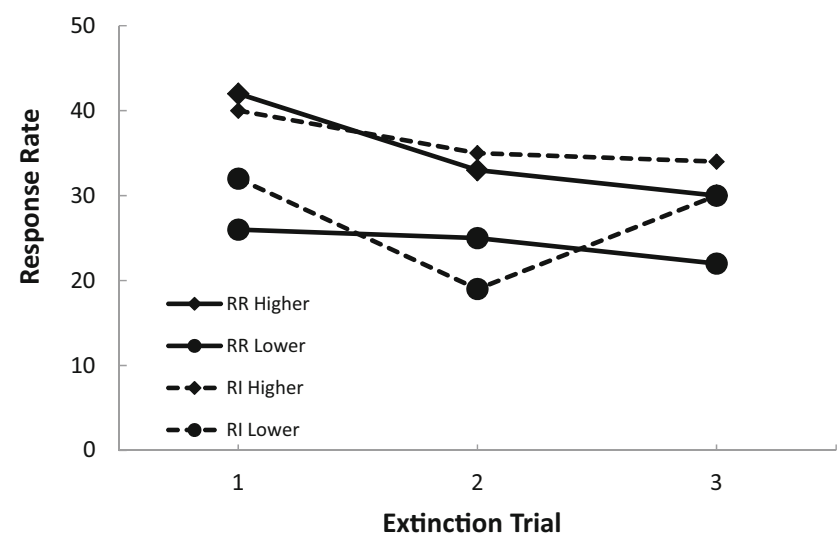

Fig. 12 Experiment 3 mean response rates per minute - RR and RI extinction trials (5-10) for higher and lower performance awareness using open-ended PA measure 
$p>.40$. Thus, on this measure the higher PA groups, measured by open-ended questionnaire, showed a larger extinction effect but only for the RR schedule.

\section{Closed-ended PA questionnaire}

Figure 13 displays the response rates for each exposure to each of the two schedule types (RR and RI) for Higher PA and Lower PA groups for the conditioning trials (trials 1-4) for the closed-ended PA questionnaire group. A three-factor, mixed-model ANOVA (PA group $\mathrm{x}$ schedule $\mathrm{x}$ trial) was conducted for the conditioning trials (trials 1-4) for this group and showed a statistically significant main effect for schedule, $F(1$, $14)=19.82, p=.001$, Partial $\mathrm{Eta}^{2}=.586$. No other significant main effects were observed, both $p \mathrm{~s}>.5$. There was a significant interaction for schedule $\mathrm{x}$ trial $\mathrm{x}$ PA group, $F(3,42)=$ $3.57, p<.05$, Partial $\mathrm{Eta}^{2}=.203$. There were no other significant interactions, $F<1$ for both. Means indicate differentiated $\mathrm{RR}$ and RI responses for the higher PA group but not for the lower PA group.

Figure 14 presents the response rates for each exposure to each of the two schedule types (RR and RI), for Higher PA and Lower PA groups for the extinction trials for the closedended PA questionnaire group. A further three-factor, mixedmodel ANOVA (schedule $\mathrm{x}$ trial $\mathrm{x}$ group) revealed a statistically significant main effects for trial, $F(2,28)=5.93, p<.07$, Partial $\mathrm{Eta}^{2}=.298$. There were no significant interactions, all $F_{\mathrm{S}}<1$. The mean rates of responding during the three extinction trials, expressed as a proportion of the mean rates of responding seen over the four training trials, produced a mean proportion of baseline responding for the lower PA group: RR $=.49( \pm .36)$ and $\mathrm{RI}=1.01( \pm .54)$; and for the higher PA group these data were $\mathrm{RR}=.38( \pm .29)$ and $\mathrm{RI}=1.02( \pm .68)$. A two-factor ANOVA (group $x$ schedule) conducted on these revealed a statically significant main effect of schedule, $F(1$, 14) $=9.64, . p<.001$, Partial $\mathrm{Eta}^{2}=.408$, but the main effect for group and the interaction were not significant, $F_{\mathrm{S}}<1$. Thus,

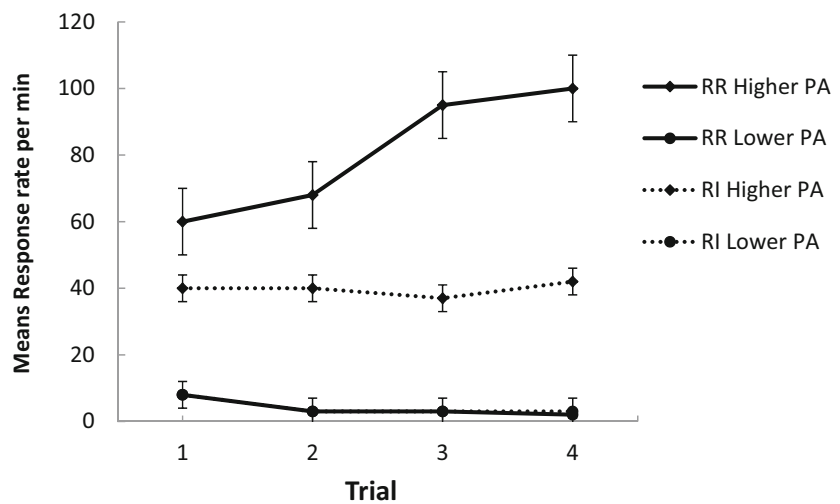

Fig. 13 Experiment 3 mean response rates per minute - RR and RI conditioning trials (1-4) for higher and lower performance awareness, using closed-ended PA measure

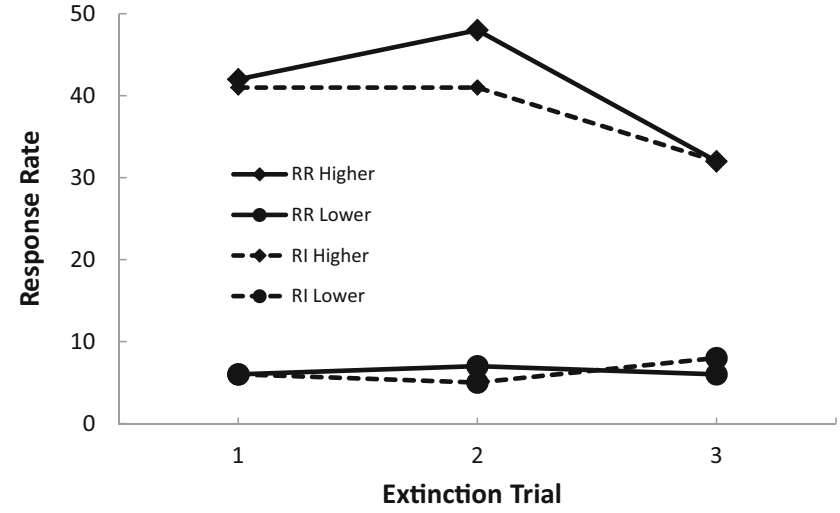

Fig. 14 Experiment 3 mean response rates per minute - RR and RI extinction trials (5-10) for higher and lower performance awareness, using closed-ended PA measure

although there was a numerical trend for greater extinction in the higher PA group for the RR schedule, as for the openended question, this was not statistically reliable.

\section{Association between instruction group and PA}

Pearson's chi-square analyses of association were conducted on the data to further investigate the relationship between performance awareness and instruction group. It was found that, for the whole sample, of the 16 participants who received minimal instructions, nine had lower performance awareness whilst seven had higher PA. Of the 16 who received accurate instructions, seven had lower PA while nine had higher PA; $\mathrm{X}^{2}$ $(1, N=32=.48, p>.7$. Thus, there was no relationship between instruction group and performance awareness in the whole sample for Experiment 3, consistent with the results of Experiments 1, and 2.

For the open-ended PA questionnaire group there were four participants in the minimal instructions group who had higher PA and four who had lower PA. Similarly for the accurate instructions group there were four with higher and four with lower PA. For the closed ended PA questionnaire group there were three participants with higher PA in the minimal instructions group and five with lower PA. For the accurate instructions group there were five with higher PA and three with lower PA. The chi-squared analyses did not yield any statistically significant results, $p \mathrm{~s}>.3$ for both.

The findings of the third experiment replicate those of experiments one and two and corroborate findings by Catania et al. (1982) and Shimoff et al. (1981), and partially corroborate findings by Hayes, et al. (1986a) in that accurate instructions were no more likely to lead to differential responding than minimal instructions. They also replicate the findings of experiments one and two in that performance awareness appeared more strongly related to differential responding according to schedule type than instruction group, similar to findings of Catania et al. (1982). 
There was no difference between either accurately versus minimally instructed participants or higher versus lower PA participants in extinction trials, suggesting that neither of these variables affects extinction. These findings are somewhat inconsistent with the findings of Experiment 2, which suggest that accurately instructed and higher PA participants both show slightly stronger extinction effects. These data also show no support for findings by Joyce and Chase (1990) and by Hayes et al. (1986a) who reported differences in extinction effects according to instruction type and performance awareness.

Furthermore, performance differentiation in relation to performance awareness did not differ significantly between the group measured by open-ended questionnaire and the group measured by closed-ended questionnaire; both groups showed differential responding in higher PA participants but not lower PA participants, suggesting that both measures are equally effective in PA measurement.

It is acknowledged that a retrospective questionnaire asking participants to think back over previous trials is flawed in some respects; however, the reliable relationship between higher PA and differentiated schedule-sensitive responses across all three experiments observable in both closed-ended and open-ended PA measures and consistent with findings using identical measures in a previous study (Bradshaw \& Reed, 2012) suggests that participants are adequately recalling their opinion of how best to score points for each trial. This may be because the trials are each reliably associated with a color which is indicated in the questions, prompting recall of color and performance. Also in the case of Experiments 2 and 3 , although participants were asked to recall 20 trials, conditioning only occurred for the first eight of these trials, which participants appeared able to recall. Nevertheless variation of the way in which PA is measured, both in terms of the questions asked and the time of measurement might be necessary to be as certain as possible of the reliability of PA measurement.

\section{General discussion}

The current research investigated the relationships between schedule responding, contingency awareness, and verbal instructions. In accordance with the results of previous studies, the findings indicated that some humans can show schedulesensitive performance on RR and RI schedules, similar to that displayed by nonhumans (see Baron \& Galizio, 1983; Bradshaw \& Reed, 2012; Matthews et al., 1977; Raia et al., 2000; Reed, 1993, 1994, 1999, 2001; Shimoff et al., 1981) and that these response patterns are related to performance awareness (see Bradshaw \& Reed, 2012).

In an earlier series of experiments, performance awareness was consistently related to schedule-sensitive responding in the sense that participants who could accurately describe RR and RI schedule contingencies performed sensitively, and those who could not describe them did not perform sensitively (Bradshaw \& Reed, 2012). Findings from this earlier study and the current study are consistent with the findings of other studies which suggest that performance awareness (most commonly achieved through shaping) results in sensitive schedule performance (see Catania et al., 1982; Hayes et al., 1986a; Matthews et al., 1985; Shimoff et al., 1981, Wearden \& Shimp, 1985). The results of the present study lend further support to the suggestion that performance awareness is an important factor in appropriate schedule responding.

The present findings suggest that although accurate experimenter-provided verbal instructions can produce sensitive schedule responding, instructions are not as important to sensitive responding as performance awareness, as demonstrated by the larger effect size for the interaction between schedule and PA than for schedule and instruction group in both experiments. Furthermore, these two factors are not related to one another; participants were no more likely to show high performance awareness for schedules having received accurate instructions on how to earn reinforcement, than they were having received minimal or inaccurate instructions.

Previous studies suggest that while verbally instructing participants on the best way to earn reinforcement generally results in schedule-sensitive responding, this responding can remain relatively rigid, showing insensitivity to either changes in the schedule contingencies or to extinction (Catania et al., 1982; Hayes et al., 1986a; Joyce \& Chase, 1990; Shimoff et al., 1981). These findings might suggest that, while participants will follow instructions given to them by experimenters, their awareness of the contingencies of the schedules is not necessarily increased by following the instructions given. Similarly, Hayes et al. (1986a) demonstrated that while accurate verbal instructions produced schedule-sensitive responding in more participants than minimal instructions, or those appropriate to either RR or RI schedules only ( $g o$ fast or go slow instructions), minimal or go fast instruction groups still demonstrated more schedule-sensitive responses than insensitive responses. Thus, instructions were not the only factor in sensitive schedule responding. The results from the present studies did not corroborate these findings as it was found that in both experiments that included extinction trials, accurately instructed participants were either just as likely to or even more likely to show extinction effects as those receiving minimal instruction.

However, it should be noted that there are procedural differences between the current experiments and those reported by Hayes et al. (1986a), which might account for this different pattern of findings. Notably, the latter study employed a much more complicated task and did not employ a response cost procedure as in the current studies. 
Shimoff et al. (1981) describe insensitivity to the schedule contingency as a defining feature of instructional control, which would suggest that participants across the three experiments described here were not actually under experimental control, as they did show sensitivity to the contingencies by extinguishing rapidly. However, Joyce and Chase (1990) showed that insensitivity to the schedule contingency might only be a feature of instructional control when that control precludes any variability of responses in the participants, which might allow them to make contact with a new contingency. One feature of our study which may have encouraged variability of responses is that all participants, regardless of instruction group, were issued with the same initial instructions that stated that it may be a good idea to respond quickly at times and slowly at other times, but that they should try to work this out for themselves. If this encouraged variability in their responses, regardless of subsequent instructions, contact with the contingencies might have been made and thus schedule sensitivity shown. Alternatively, our findings may be related to the relatively simplistic schedules employed, which made it easier to judge that reinforcement was no longer available.

Hayes et al. (1986a) discuss the fact that variability of responses does not provide a complete explanation for differentiated behavior because some participants in their research made contact with contingencies that opposed the rule provided and yet still followed that rule. Furthermore they argue that a change of contingency via extinction is enough to constitute contact with the contingency in itself and, thus, to some extent, all participants contact the contingency to some degree. They reject the claim by Shimoff et al. (1981) that insensitivity to the schedule contingency is a defining feature of instructional control on the grounds that this would mean that those showing initial adherence to instructions but then also showing sensitivity to the contingency could not be described as having been under instructional control. They argue that adhering to the instructions for a period of time is showing at least partial instructional control. The results of our experiments suggest that participants may not have been under full instructional control due to the lack of differentiated responding between RR and RI schedules for those in the accurate instructions groups in Experiments 2 and 3. Two features of the current study might have produced this finding: all participants in both instruction groups received the same instructions to vary their responses initially before later minimal or accurate instructions; and participants were not instructed as to which stimulus color would indicate the need for slow or rapid pressing. Replication of the study with instructions varied further to include or exclude initial instructions to vary their responses and more specific instructions to press rapidly when the stimulus box was red for example, and slowly when green might produce results which demonstrate instructional control observable in the data.
This study measured performance awareness in terms of asking participants what they thought was the best way to score points. Findings by Matthews et al. (1985) suggested that measurement of performance awareness might produce more reliable associations between verbal descriptions and responding and between higher PA and schedule-sensitive responding than contingency awareness measurement. This might be because it is easier for participants to describe a task in terms of what they did rather than what the task required. Differentiation between performance awareness and contingency awareness seems to be an area for further investigation following the results of this experiment and the difficulties with precise performance awareness measurement.

The results of this study and a previous study (Bradshaw \& Reed, 2012) suggest that performance awareness is a pivotal factor in the analysis of schedule responding in humans, but they do not address the wider question of what PA is and why it is so important. It may not be a uniquely human characteristic, even though it has been demonstrated to strongly relate to human differentiated schedule performance which differs from that of animals, since some studies have shown a degree of contingency awareness in nonhuman animals (e.g., see Lattal, 1975). It would seem that some investigation into the nature of PA and/or CA and further exploration of its relationship with human schedule performance is a necessity following the present results. Awareness might be an implicit, verbal process that is unique to humans or which is common to many species. Or it might be better described as a verbal, selfgenerated process that takes on greater importance than rules imposed by others. Future research might seek to explore the nature of this awareness and might also vary the nature of reinforcement provided and the method of measurement of PA and CA as a means to further investigate this important concern.

\section{References}

Baeyens, F., Eelen, P., \& Van den Bergh, O. (1990). Contingency awareness in evaluative conditioning: A case for unaware affective-evaluative learning. Cognition \& Emotion, 4, 3-18.

Baron, A., \& Galizio, M. (1983). Instructional control of human operant behaviour. The Psychological Record, 33, 495-520.

Bradshaw, C. A., \& Reed, P. (2012). Relationship between contingency awareness and human performance on random ratio and random interval schedules. Learning and Motivation, 43, 55-65.

Brewer, W. F. (1974). No convincing evidence for conditioning in adult humans. In W. B. Weimer \& D. S. Palermo (Eds.), Cognition and the symbolic processes (pp. 1-42). Hillsdale: Erlbaum.

Catania, A. C., Matthews, T. J., Silverman, P. J., \& Yohalem, R. (1977). Yoked variable-ratio and variable-interval responding in pigeons. Journal of the Experimental Analysis of Behavior, 28, 155-161.

Catania, A. C., Matthews, B. A., \& Shimoff, E. (1982). Instructed versus shaped verbal human behavior: Interactions with nonverbal 
responding. Journal of the Experimental Analysis of Behavior, 38, 233-248.

Catania, A. C., \& Reynolds, G. S. (1968). A quantitative analysis of the responding maintained by interval schedules of reinforcement. Journal of the Experimental Analysis of Behavior, 11, 327-383.

Cerutti, D. T. (1989). Discrimination theory of rule-governed behavior. Journal of the Experimental Analysis of Behavior, 51(2), 259-276.

Dawson, M. E., \& Schell, A. M. (1985). Information processing and human autonomic classical conditioning. In P. K. Ackles, J. R. Jennings, \& M. Coles (Eds.), Advances in psychophysiology (Vol. 1). Greenwich: JAI Press.

Dickinson, A., \& Brown, K. J. (2007). Flavor-evaluative conditioning is unaffected by contingency knowledge during training with colorflavor compounds. Learning \& Behavior, 35, 36-42.

Ferster, C. B., \& Skinner, B. F. (1957). Schedules of reinforcement. New York: Appleton-Century-Crofts.

Galizio, M. (1979). Contingency-shaped and rule-governd behavior: Instructional control of human loss avoidance. Journal of the Experimental Analysis of Behavior, 31(1), 53-70.

Hayes, S. C., Brownstein, A. J., Haas, J. R., \& Greenway, D. E. (1986a). Instructions, multiple schedules, and extinction: Distinguishing rulegoverned from schedule-controlled behavior. Journal of the Experimental Analysis of Behavior, 46(2), 137-147.

Hayes, S. C., Brownstein, A. J., Zettle, R. D., Rosenfarb, I., \& Korn, Z. (1986b). Rule-governed behavior and sensitivity to changing consequences of responding. Journal of the Experimental Analysis of Behavior, 45, 237-256.

Hofmann, W., De Houwer, J., Perugini, M., Baeyens, F., \& Crombez, G. (2010). Evaluative conditioning in humans: A meta-analysis. Psychological Bulletin, 136, 390-421.

Joyce, J. H., \& Chase, P. N. (1990). Effects of response variability on the sensitivity of rule-governed behavior. Journal of the Experimental Analysis of Behavior, 54, 251-262.

Lattal, K. A. (1975). Reinforcement contingencies as discriminative stimuli. Journal of the Experimental Analysis of Behavior, 23, 241-246.

Leander, J. D., Lippman, L. G., \& Meyer, M. M. (1968). Fixed interval performance as related to subjects' verbalizations of the reinforcement contingency. The Psychological Record, 18, 469-474.

Lippman, L. G., \& Meyer, M. M. (1967). Fixed interval performance as related to instructions and to subjects' verbalization of the contingency. Psychonomic Science, 8, 135-136.

Lowe, C. F. (1979). Determinants of human operant behavior. In M. D. Zeiler \& P. Harzem (Eds.), Advances in analysis of behaviour (Reinforcement and the organisation of behaviour, Vol. 1, pp. 7193). Chichester: Wiley.

Lowe, C. F., Harzem, P., \& Bagshaw, M. (1978a). Species differences in temporal control of behavior. II: Human performance. Journal of the Experimental Analysis of Behavior, 29, 351-361.

Lowe, C. F., Harzem, P., \& Hughes, S. (1978b). Determinants of operant behaviour in humans: Some differences from animals. Quarterly Journal of Experimental Psychology, 30, 373-386.

Matthews, B. A., Catania, A. C., \& Shimoff, E. (1985). Effects of uninstructed verbal behavior on nonverbal responding: Contingency description versus performance descriptions. Journal of the Experimental Analysis of Behavior, 43, 155-164.

Matthews, B. A., Shimoff, E., Catania, A. C., \& Sagvolden, T. (1977). Uninstructed human responding: Sensitivity to ratio and interval contingencies. Journal of the Experimental Analysis of Behavior, 27, 453-467.

Mitchell, C. J., De Houwer, J., \& Lovibond, P. F. (2009). The propositional nature of human associative learning. Behavioral and Brain Sciences, 32, 183-198.

Osborne, L. A., \& Reed, P. (2008). Parents' perceptions of communication with professionals during the diagnosis of autism. Autism, 12(3), 309-324.
Peele, D. B., Casey, J., \& Silverberg, A. (1984). Primacy of interresponsetime reinforcement accounting for rates under variable-ratio and variable-interval schedules. Journal of Experimental Psychology: Animal Behavior Processes, 10, 149-167.

Pleyers, G., Corneille, O., Luminet, O., \& Yzerbyt, V. (2007). Aware and (dis)liking: Item-based analyses reveal that valence acquisition via evaluative conditioning emerges only when there is contingency awareness. Journal of Experimental Psychology: Learning, Memory, and Cognition, 33, 130-144.

Raia, C. P., Shillingford, S. A., Miller, H. L., \& Baier, P. S. (2000). Interaction in procedural factors in human performance on yoked schedules. Journal of the Experimental Analysis of Behavior, 74, 265-281.

Reed, P. (1993). Influence of schedule of outcome presentation on human judgments of causality. Quarterly Journal of Experimental Psychology, 46A, 327-345.

Reed, P. (1994). Influence of the cost of responding on human causality judgments. Memory \& Cognition, 22, 243-248.

Reed, P. (1999). Effect of perceived cost on judgments regarding the efficacy of investment. Journal of Economic Psychology, 20, 657676.

Reed, P. (2001). Schedules of reinforcement as determinants of human causality judgments and response rates. Journal of Experimental Psychology: Animal Behavior Processes, 27, 187-195.

Reed, P. (2011). An experimental analysis of steady-state response rate components on variable ratio and variable interval schedules of reinforcement. Journal of Experimental Psychology: Animal Behavior Processes, 37, 1-9.

Reynolds, G. S. (1975). A Primer of operant conditioning. Glenview: Scott Foresman.

Shimoff, E., Catania, A. C., \& Matthews, B. A. (1981). Uninstructed human responding: Responsivity of low-rate performance to schedule contingencies. Journal of the Experimental Analysis of Behaviour, 36, 207-220.

Shimoff, E., Matthews, B. A., \& Catania, A. C. (1986). Human operant performance: Sensitivity and pseudosensitivity to contingencies. Journal of the Experimental Analysis of Behavior, 46, 149-157.

Stahl, C., \& Unkelbach, C. (2009). Evaluative learning with single versus multiple USs: The role of contingency awareness. Journal of Experimental Psychology: Animal Behavior Processes, 35, 286291.

Stahl, C., Unkelbach, C., \& Corneille, O. (2009). On the respective contributions of awareness of US valence and US identity in valence acquisition through evaluative conditioning. Journal of Personality and Social Psychology, 97, 404-420.

Vaughn, S., Schumm, J. S., \& Sinagub, J. M. (1996). Focus group interviews in education and psychology. London: Sage Publications.

Walther, E., \& Nagengast, B. (2006). Evaluative conditioning and the awareness issue: Assessing contingency awareness with the fourpicture recognition test. Journal of Experimental Psychology: Animal Behavior Processes, 32, 454-459.

Wearden, J. H., \& Shimp, C. P. (1985). Local temporal patterning of operant behavior in humans. Journal of the Experimental Analysis of Behavior, 44, 315-324.

Weiner, H. (1964). Conditioning history and human fixed-interval performance. Journal of the Experimental Analysis of Behavior, 7, 383385.

Weiner, H. (1969). Controlling human fixed-interval performance. Journal of the Experimental Analysis of Behavior, 12, 349-373.

Weiner, H. (1970). Human behavioural persistence. Psychological Record, 20, 445-446.

Zuriff, G. E. (1970). A comparison of variable-ratio and variable-interval schedules of reinforcement. Journal of the Experimental Analysis of Behavior, 13, 369-374. 